\title{
Predictability and number of pairings in Pavlovian fear conditioning'
}

ROBERT A. RESCORLA ${ }^{2}$

UNIVERSITY OF PENNSYLVANIA

Three groups of dogs were Sidman avoidance trained. They then received different kinds of Pavlovian fear conditioning. For one group CSs and USs occurred randomly and independently; for a second group, CSs predicted the occurrence of USs; for a third group, CSs predicted the absence of the USs. The CSs were subsequently presented while $S$ performed the avoidance response. CSs which had predicted the occurrence or the absence of USs produced, respectively, increases and decreases in avoidance rate. For the group with random $C S s$ and USs in conditioning, the CS had no effect upon avoidance.

Traditional conceptions of Pavlovian conditioning have emphasized the pairing of CS and US as the essential condition for the development of a CR. As long as the CS and US occur in temporal contiguity, the conditions for Pavlovian conditioning are assumed to be met. In contrast, another view of Pavlovian conditioning argues that conditioning depends upon the degree to which the CS allows $S$ to predict the occurrence of the US. If the CS is followed by a change in the probability of the US, Pavlovian conditioning will occur. If the CS forecasts an increased likelihood of the US, excitatory conditioning will occur; if the CS forecasts a decreased likelihood of the US, the CS will take on inhibitory properties. According to this view, the number of CS-US pairings may be irrelevant to the development of a CR if the CS does not predict a change in the probability of occurrence of the US.

The experiment reported here explores the fruitfulness of this second approach to Pavlovian fear conditioning. Three groups of dogs received different kinds of Pavlovian conditioning. For one group, CSs and USs occurred randomly and independently in such a way that CS occurrences provided no information about US occurrences. In a second group, CS occurrences were followed by an increase in the probability of US occurrences; however, $\mathrm{Ss}$ in this group received the same number of CS-US pairings as did Ss in the first group. For a third group, CS occurrences predicted the absence of USs. These CSs were there presented while $S$ performed a previously trained avoidance response. Increases in the rate of avoidance responding produced by CSs were taken as evidence for excitatory fear conditioning and decreases were taken as indicating inhibition of fear. Such changes in rate of avoidance responding have been shown by Rescorla \& LoLordo (1965) to be a sensitive index of the level of conditioned fear.

Method

Ss were 18 mongrel dogs, individually housed and maintained on ad lib food and water throughout the experiment. The apparatus was a two-compartment dog shuttlebox described in detail by Solomon \& Wynne (1953). The two compartments were separated by a barrier of adjustable height and by a drop gate which, when lowered, prevented $S$ from crossing from one compartment into the other. The floor was composed of stainless steel grids which could be electrified through a scrambler. Speakers, mounted above the hardware-cloth ceiling, provided a contiruous white noise background and permitted the presentation of tonal stimuli.

The training procedure was similar to that described by Rescorla \& LoLordo (1965). Each S was trained to jump the barrier, separating the two sides of the shuttlebox, to avoid electric shock. Brief shocks, 0.25 sec., were programmed on a Sidman avoidance schedule; the shock-shock interval was $10 \mathrm{sec}$. and the responseshock interval was $30 \mathrm{sec}$. The Ss received three initial days of avoidance training. On the first day the barrier height was 9 in. and the shock level $6 \mathrm{ma}$; on all subsequent days, the barrier height was 15 in. and the shock set at $8 \mathrm{ma}$.

Beginning with the fourth experimental day, $S$ was confined to one-half of the shuttlebox and given Pavlovian fear conditioning. For the sixdogs in Group $\mathbf{R}$ (random), 24, 5-sec., 3 ma shocks were programmed on a variable interval schedule with a mean of $2.5 \mathrm{~min}$. Twenty-four, 5-sec., $400 \mathrm{cps}$ tones were independently programmed randomly throughout the session in such a way that a tone onset was equiprobable at any time in the session. This was accomplished by a VI timer and a series of tapes. The six dogs in Group $P$ (positive prediction) received a treatment identical to that of Group $R$ except that they received only those shocks which were programmed to occur during the $30 \mathrm{sec}$. following each tone onset. The six dogs in Group $\mathrm{N}$ (negative prediction) received a treatment identical to that of Group $R$ except that they received only those shocks which were not programmed to occur within $30 \mathrm{sec}$. after a tone onset. The treatments for Groups $P$ and $N$ were accomplished by having each CS onset reset a $30 \mathrm{sec}$. timer through which the pre-programmed shocks were gated. Thus, for Group P, CS occurrences predicted US occurrences; and for Group N, CS occurrences predicted absence of USs.

Pavlovian conditioning and Sidman avoidance training days were then alternated until $S$ had received a total of seven avoidance and five conditioning sessions. On day 13 a single test session was given. During this session, $\mathrm{S}$ performed the avoidance response with the Sidman schedule remaining in effect. In addition, 24, 5-sec., 400 cps tones were superimposed upon the avoidance behavior with a mean intertrial interval of $2.5 \mathrm{~min}$. 
Changes in the rate of avoidance induced by these CSs were used as an index of the conditioned excitatory and inhibitory effects of the tones.

\section{Results}

The Sidman avoidance response was rapidly acquired by most animals and after several sessions all Ss were reliable responders. Figure 1 shows the results of the test session. Plotted in this figure are the mean number of responses per 5-sec. period of time over successive 5-sec. periods. Prior to the occurrence of a CS, all groups responded at approximately the same rate. However, the occurrence of a CS led to markedly different results in the three groups. For Group P, CS onset produced an abrupt increase in response rate followed by a return to base rate. The rate increase was confined to the first few 5-sec. periods following CS onset. In contrast, the CS produced a sharp decrease in rate in Group N. Again the rate change was maximal immediately following $\mathrm{CS}$ onset. For Group $\mathbf{R}$, the occurrence of a CS produced very little effect.

Comparisons among the groups were made with the help of suppression ratios. These ratios are of the form $A /(A+B)$ where $B$ is the mean rate in the 30 sec. prior to $C S$ onset and $A$ is the rate for the period on which the two groups are to be compared. Using this measure, the rate increase during the CS was reliably greater for Group $P$ than for Group $R(U=0 ; p<.01)$. Group $R$, in turn, responded more frequently during $C S$ than did Group N $(U=0 ; p<.01)$. Similar conclusions result if the groups are compared on the rate during the entire $30 \mathrm{sec}$. following CS onset.

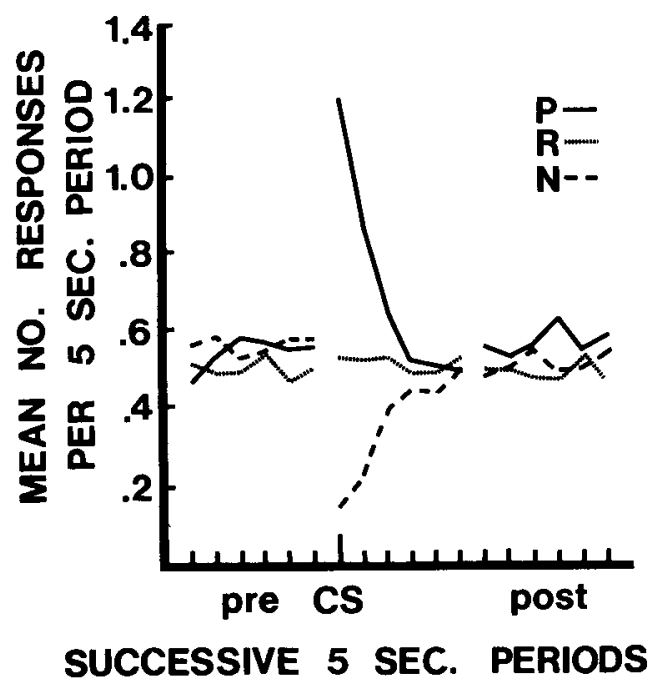

Fig. 1. Mean number of responses per 5-sec. period in successive periods prior to $\mathrm{CS}$ onset, during the $\mathrm{CS}$ and the subsequent 25 sec. of differential conditioning treatment, and after the expiration of the 25 sec. period.

\section{Discussion}

The results of this experiment indicate that the degree to which a CS allows S to predict US occurrences is an important variable in Pavlovian fear conditioning. Stimuli which signalled increased probability of the US became elicitors of fear, resulting in an increased jumping rate, and stimuli which signalled decreased probability of the US became inhibitors of fear, resulting in a decreased jumping rate. The results, therefore, substantiate the findings of Rescorla \& LoLordo (1965), that active inhibition and excitation of fear can be induced by Pavlovian methods. However, these effects seem to be independent of the more traditionally emphasized effects of number of CS-US pairings. Despite the fact that $\mathrm{Ss}$ in Group $R$ received at least as many pairings of the CS and US as Ss in Group P, only the Ss in Group $P$ showed evidence of Pavlovian fear conditioning.

The temporal location of the effect produced by the CS is also of interest. The differential effects of the CS for the three groups were primarily confined to the periods immediately following CS onset. Perhaps this happened because shocks were uniformly distributed, and for Group $P$ the probability of a US in the next $30 \mathrm{sec}$. was maximal just after CS onset and declined as time since the CS increased; but for Group $\mathrm{N}$, the probability of a shock was minimal immediately after CSonset. Another possibility is that the period immediately after CSonset is simply more discriminable from the baseline conditions than are subsequent periods.

These results suggest that we consider as a basic dimension of Pavlovian conditioning the degree to which the US is contingent upon prior CSs. From this point of view, the appropriate control procedure for nonassociative effects of Pavlovian conditioning, such as sensitization or pseudoconditioning, is one in which there is no contingency between CS and US. The two extremes in which CS predicts either the increased or the decreased probability of a US are seen in the present experiment to produce, respectively, excitation and inhibition. A procedure such as that of Group $R$ in which there is no contingency between CS and US provides an appropriate control procedure against which to evaluate both of these effects.

\section{References}

Rescorla, R. A., \& LoLondo, V. M. Inhibition of avoidance behavior. J. comp. physiol. Psychol., 1965, 59, 406-412.

Solomon, R. L., \& Wynne, L. C. Traumatic avoidance learning: Acquisition in normal dogs. Psychol. Monogr., 1953, 67, No. 4 (Whole No. 354).

\section{Notes}

1. NSF predoctoral fellow.

2. This research was supported by United States Public Health Service Grant MH-04202 to Richard L. Solomon. The author thanks Dr. Solomon for his advice and criticism. 